\title{
Introduction
}

\section{Advanced Manufacturing for Energy and Transportation International School}

\author{
Nihed CHAÂBANE ${ }^{1}$ and Frédéric SCHUSTER ${ }^{2}$ \\ ${ }^{1}$ Université Paris Saclay, CEA, Institut National des Sciences et Techniques \\ Nucléaires, 91191 Gif-sur-Yvette, France \\ ${ }^{2}$ Université Paris Saclay, CEA, Cross-cutting Program on Materials Science \\ and Engineering, 91191 Gif-sur-Yvette, France
}

\begin{abstract}
The objective of Advanced Manufacturing for Energy and Transportation International School (AMETIS) is to present an integrated vision of advanced manufacturing dedicated to materials and components for low-carbon energy and transportation. The proposed approach essentially focuses on three main families of emerging processes, as well as their possible synergies to generate an often disruptive innovation framework. The option is also to present new methodologies that allow faster discovery or design of new materials of interest or faster optimization of complex emerging processes. These methodologies are often based on the significant progress made by digital technologies that have a considerable impact on the acceleration of Materials Science and Engineering. In particular, they make it possible to considerably shorten development time and minimize costs. Finally, this approach cannot be global without taking into account the drivers of sustainability in terms of resources, environment, safety and society. Therefore, AMETIS proposes a holistic approach.
\end{abstract}

\subsection{Introduction}

The impact of Materials Science and Engineering on innovation in the field of components for low-carbon energy and transportation is considerable. Issues related to the durability of solutions, such as economic sustainability, are often strongly linked to the choice of materials and their production process, which have a very important impact on performance during use all along the lifetime of the 
components. Therefore, the advanced manufacturing concept integrates the entire value chain, from eco-design to recycling, and even upcycling, taking into account a number of environmental, social and resource management factors including energy sobriety. This systemic presentation is the core of the AMETIS (figure 1).

Among the large families of emerging processes, AMETIS focuses on three of them due to the high potential of each one, and it is also their possible synergies that can initiate breakthrough innovations or incremental advances, as shown in figure 2 .

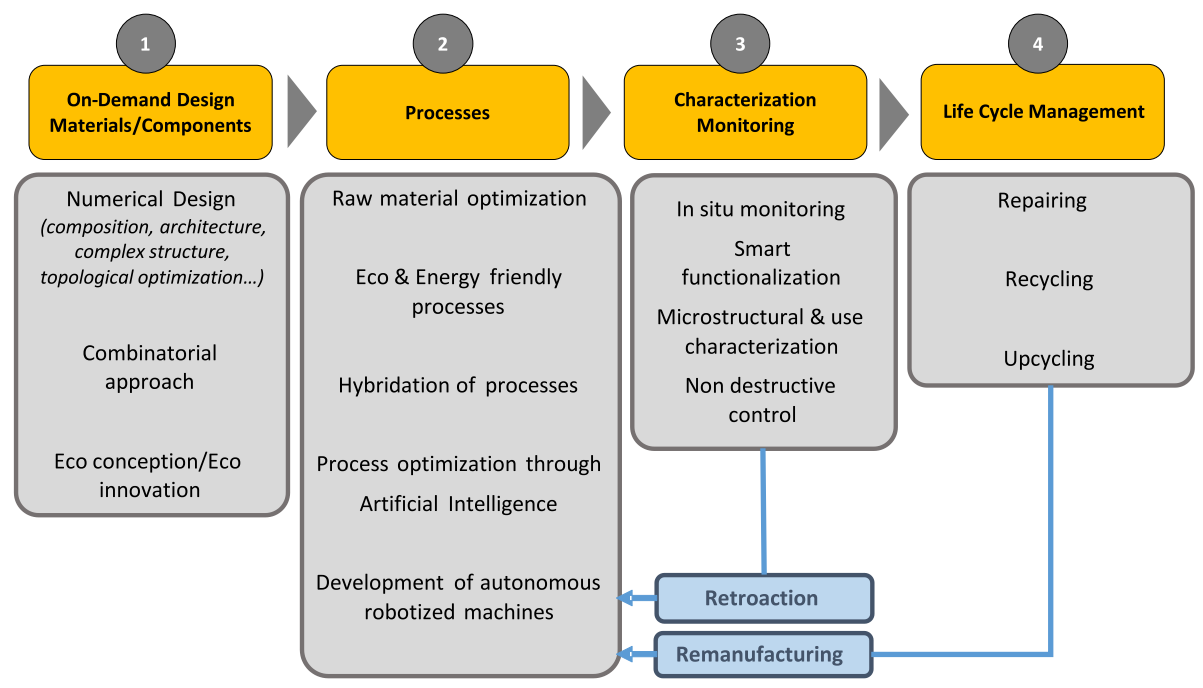

FIG. 1 - Systemic approach of advanced manufacturing.

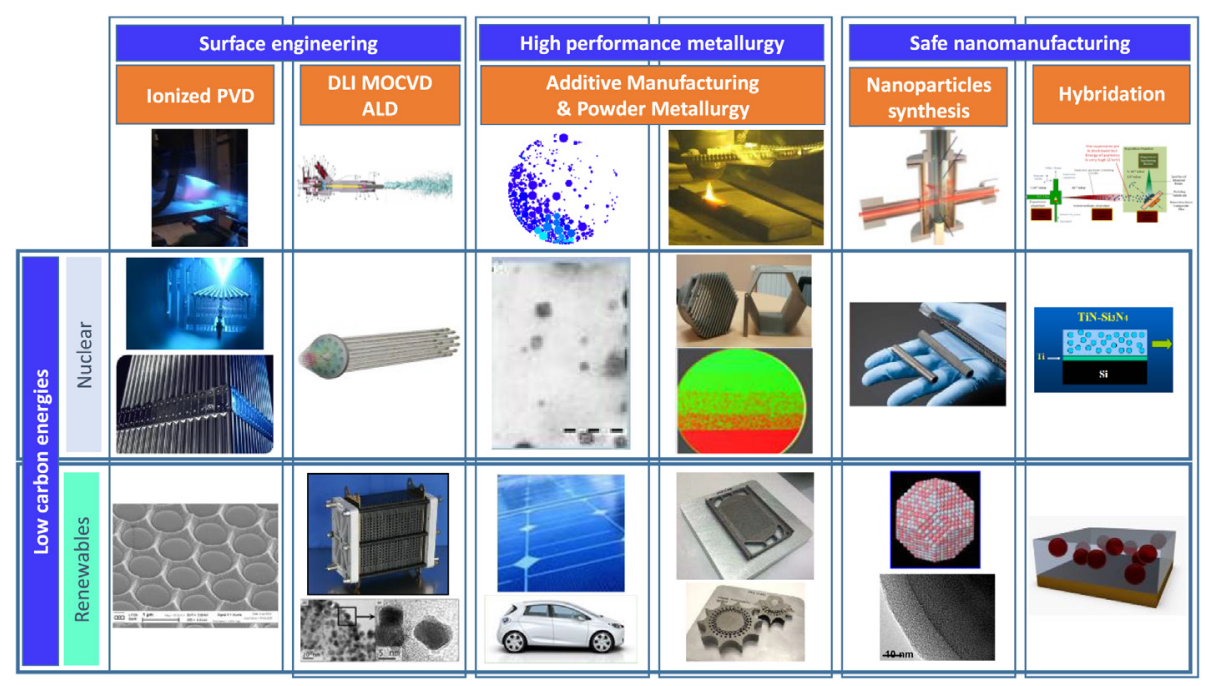

FIG. 2 - Examples of emerging processes for low carbon energies. 
Therefore, after presenting an integrated vision of additive manufacturing, the latest advancements in surface engineering and nanomanufacturing technologies, AMETIS will focus on the convergence of these technologies as a source of innovation for advanced energy manufacturing and transport.

\subsection{Holistic Overview of Additive Manufacturing}

Regarding additive manufacturing, AMETIS will first give a presentation of an integrated vision of this family of technologies, presenting in particular the different processes, the entire value chain, the different types of materials processed, as well as examples in particular in the field of transport and low carbon energies. Then, AMETIS will focus on several trends that are generally not presented in technology-only instruction. AMETIS will first illustrate the relationships between process, microstructure and properties and also the importance of mastering raw materials from the start to the end result in terms of product performance. Next, AMETIS will present the design of on-demand architectures according to the required properties. This approach is based on digital design in a first step and the synthesis of these architectures through additive manufacturing in a second step, to compare reality with prediction, thus illustrating the link that can be established between numerical design and manufacturing of on-demand high-performance materials and components. Finally, AMETIS will focus on the possible smart integration of the electronic functions "Package Electronics Additive Manufacturing" that allows the technology to enter the era of the Internet of Things, with, for example, the possibility of monitoring the performance of the components remotely and their durability during the life time.

\subsection{Integrated Approach of Nanomanufacturing: From Nanoobject Synthesis to Applications}

The second "pillar process", also with a very generic character, consists of nanofabrication. In fact, the synthesis and safe integration of nanoobjects (nanopowders, nanowires, and nanotubes) into components for energy and transport is a fairly unique way to develop innovation in these sectors. AMETIS will present the main technologies for the development and implementation of nanoobjects, as well as the resulting applications. In addition to these purely scientific and technological aspects, the contribution of nanotechnologies to the development of sustainable solutions will also be presented. In fact, in some cases, nanotechnologies can lead to a reduction in the use of critical metals or strategic materials, and sometimes even allow their replacement. The last, but not least, consideration will be devoted to managing risks using nanomaterials as part of an integrated approach from production to consumer. 


\subsection{Emerging Surface Engineering Processes}

Surface engineering has made remarkable progress in the past decade and new applications, particularly in the energy field, have become possible and are currently under industrial development in part due to this progress. Following a presentation based on the principles of thin films and thermal spray technologies, AMETIS will focus on a more detailed presentation of the progress made in recent years. In the Physical Vapor Deposition (PVD) sector, the development of highly ionized technologies and, in particular, HIPIMS (High Power Impulse Magnetron Sputtering) technology has enabled significant progress to be made, for example, in nuclear applications (EATF: Enhanced Accident Tolerant Fuel and also in the reprocessing sector). In terms of Chemical Vapor Deposition (CVD), the penetration of technologies such as DLI-MOCVD (Direct Liquid Injection MOCVD) and ALD (Atomic Layer Deposition) in the low carbon energy sector exploits all the richness and diversity of the chemistry of organometallic precursors, both for applications in extreme environments and for functional components for batteries, supercapacitors and high-temperature electrolyzers or fuel cell components. Regarding thermal spraying technologies, two very important advances are observed, firstly, the significant development of cold spraying technology, which can also be considered in some aspects as a repair or additive manufacturing technology. Another important innovation refers to the thermal plasma spraying of nanoparticle precursor solutions or suspensions that offers very interesting perspectives for the new generations of thermal and environmental barriers, in particular for the aeronautical and space sector.

\subsection{Possible Synergies Between Technologies}

After the presentation of the three main families of emerging processes, AMETIS will also present their possible synergies and the resulting innovations (figure 3), in particular:

- At the interfaces between 2D and 3D processes

This is usually the case when, for example, 3D manufacturing processes are combined with surface functionalization processes that give these architectures new functionalities (catalytic, anti-corrosion, electrical, etc.).

- At the border between 3D printing and nanofabrication

This is, for example, the case of the use of nanoparticles as raw material for 3D printing technologies, or for the in situ generation of nanocomposite materials in additive manufacturing machines (in situ generation of ODS (Oxide dispersion strengthened) steels, for example).

- With a combination of nanotechnologies and surface engineering 


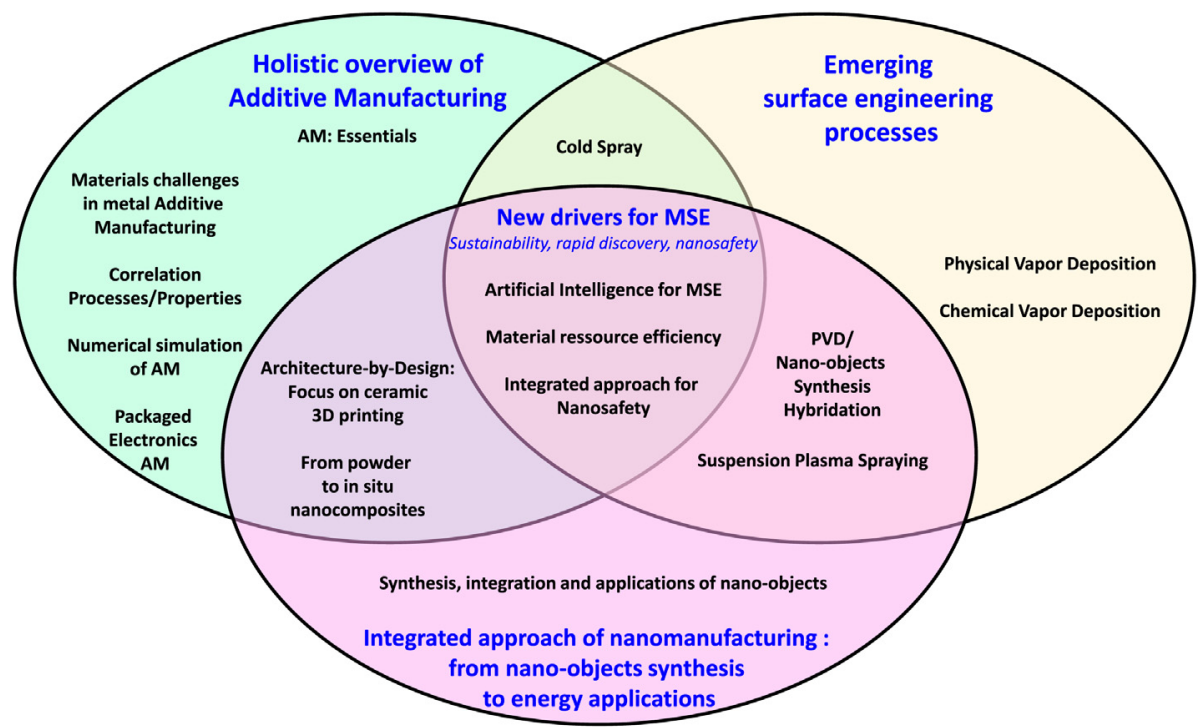

FIG. 3 - The three families of emerging processes, their possible synergies, the new generic methodologies for rapid discovery and optimization and the new drivers for MSE.

It is a completely new generation of innovative processes that combine thin film science and engineering with nanoparticle synthesis technologies. This new approach enables the production of innovative nanocomposite materials, in which the choice of the nature of the matrix is completely independent of the nature of the incorporated nanoobjects, which greatly opens the spectrum of applications for this type of new materials (solar thermal energy, photovoltaic energy, batteries...).

\subsection{New Methodologies for Rapid Discovery of Materials and New Drivers for MSE}

These three major families of processes also contribute to an accelerated discovery of new materials, components and solutions, either because they often intrinsically allow rapid screening of compositions, microstructures and architectures, or because they give access to new concepts of materials and components hitherto not accessible through more conventional production routes.

This accelerated discovery of solutions also benefits from considerable progress in digital technologies, both for data mining, numerical design of materials, components and architectures, numerical simulation of processes, as well as their optimization using Artificial Intelligence approaches. These new approaches are also intended to be introduced during this first AMETIS. 
The final viewpoint to address in this systemic overview of advanced manufacturing is about the new drivers for the development of a sustainable Materials Science and Engineering. These drivers consist in environmental and energy sobriety of the processes, minimization of the use of resources, in particular of strategic metals and, in general, in the impact of emerging processes on the circular economy of materials and recyclability of components. Therefore, the objective of this first summer school on advanced manufacturing is to offer a systemic approach taking into account the drivers of sustainability of the implemented solutions and eco-innovation. In more general terms, this systemic approach and these new convergences are the core business of the scientific project of the IMPACT International Chair of INSTN (National Institute of Nuclear Science and Technology) at the University of Paris-Saclay (https://www.materials-impact-chair.org/).

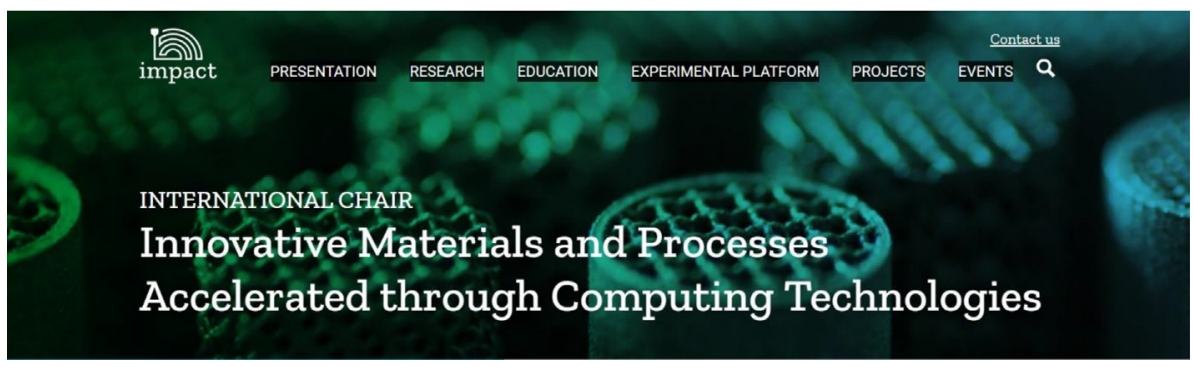

